Recepción: 23 / 02 / 2018

Aceptación: 18 / 04 / 2018

Publicación: 05 / 07 / 2018

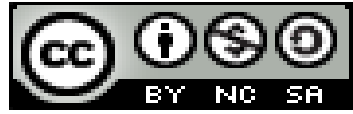

Ciencias de la Salud

Artículo de Investigación

\title{
Identidad y competencias ocupacionales pre laboral en usuarios de la fundación Sergio Plaza Aguas con discapacidad intelectual leve
}

Identity and pre-employment occupational competencies in users of the Sergio Plaza Foundation Waters with mild intellectual disability

Identidade e competências ocupacionais pré-emprego em usuários da Fundação Sergio Plaza Águas com deficiência intelectual leve

\author{
Macrina N. Bueno-Pinargote ${ }^{\mathrm{I}}$ \\ nataliabuenop-15@hotmail.com \\ Jhonny P. Palacios-Martinez ${ }^{\text {II }}$ \\ lcdopalaciospat@hotmail.com \\ María J. Loor-Zamora III \\ majoskr@hotmail.com
}

Correspondencia: nataliabuenop-15@hotmail.com

\footnotetext{
I Licenciada en Terapia Ocupacional, Docente de la Universidad de Guayaquil; Guayaquil, Ecuador.

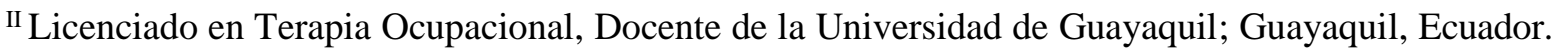

III Licenciada en Terapia Ocupacional, Docente de la Universidad de Guayaquil; Guayaquil,Ecuador.
} 


\section{Resumen}

La discapacidad intelectual leve es una condición que se presenta en varios tipos de discapacidades o de manera absoluta. La problemática de la discapacidad intelectual en el Ecuador se da debido a que el mayor porcentaje es de familias que se convierten en ayuda directa para la persona con Discapacidad en lo que en competencia e identidad ocupacional se refiere. Al respecto, en este artículo se evaluó la identidad y competencias ocupacionales en usuarios que asisten a la fundación Sergio Plaza Aguas con discapacidad intelectual leve. La metodología fue bajo un enfoque cuali-cuantitativo apoyado en un proyecto viable y factible de tipo descriptivo experimental y de corte transversal. La muestra estuvo constituida por 32 usuarios y la técnica para la recolección de la información fue la observación. Los resultados determinaron que inicialmente el $22 \%$ de los usuarios presentaron problemas de identidad y de competencias por alguna ocupación y posterior a la evaluación final descendió a 16\% en ambos casos favoreciendo la evaluación. Entre sus conclusiones, se pudo determinar que la aplicación de un plan de intervención ocupacional mejora la identidad y competencias ocupacionales en usuarios con discapacidad intelectual leve.

Palabras clave: identidad; competencias ocupacionales; discapacidad intelectual leve y plan de intervención.

\section{Abstract}

Mild intellectual disability is a condition that occurs in various types of disabilities or absolutely. The problem of intellectual disability in Ecuador is due to the fact that the highest percentage is of families that become direct help for the person with Disability in terms of competence and occupational identity. In this regard, this article evaluated the identity and occupational skills of users who attend the Sergio Plaza Aguas Foundation with mild intellectual disability. The methodology was based on a qualitative and quantitative approach supported by a feasible and feasible project of an experimental and cross-sectional descriptive type. The sample consisted of 32 users and the technique for collecting the information was observation. The results determined that initially $22 \%$ of the users presented problems of identity and competences for some occupation and after the final evaluation it decreased to $16 \%$ in both cases favoring the evaluation. Among its conclusions, it was determined that the application of an occupational 
intervention plan improves the identity and occupational competencies of users with mild intellectual disability.

Keywords: identity, occupational skills, mild intellectual disability and intervention plan.

\section{Resumo}

A deficiência intelectual leve é uma condição que ocorre em vários tipos de deficiência ou absolutamente. O problema da deficiência intelectual no Equador deve-se ao fato de que o maior percentual é de famílias que se tornam ajuda direta para a pessoa com Deficiência em termos de competência e identidade ocupacional. Nesse sentido, este artigo avaliou as habilidades identitárias e ocupacionais dos usuários que frequentam a Fundação Sergio Plaza Aguas com deficiência intelectual leve. A metodologia baseou-se em uma abordagem qualitativa e quantitativa, apoiada em um projeto viável e viável, do tipo descritivo experimental e transversal. A amostra foi composta por 32 usuários e a técnica de coleta das informações foi a observação. Os resultados determinaram que inicialmente $22 \%$ dos usuários apresentavam problemas de identidade e competências para alguma ocupação e após a avaliação final diminuiu para $16 \%$ em ambos os casos favorecendo a avaliação. Entre suas conclusões, determinou-se que a aplicação de um plano de intervenção ocupacional melhora a identidade e as competências ocupacionais dos usuários com deficiência mental leve.

Palavras chave: identidade, habilidades ocupacionais, deficiência intelectual leve e plano de intervenção.

\section{Introducción}

El termino Discapacidad intelectual se utiliza cuando existe presencia de un desarrollo mental incompleto o detenido, caracterizado principalmente por el deterioro de funciones específicas tales como: cognoscitivas, del lenguaje, motrices y socialización perteneciente a cada etapa del desarrollo y que contribuyen al nivel global de inteligencia. Según el Consejo Nacional de Discapacidades (CONADIS), Perú es el país que presenta mayor porcentaje de discapacitados con 18,5\%; seguido de Estados Unidos con $15 \%$ y finalmente Ecuador con 12,8\%. En la actualidad Ecuador cuenta con 1'653.000 personas con discapacidad registradas en el último censo. 
Por otro lado, la discapacidad intelectual, para Bassedas (2010), la define como "La discapacidad intelectual se refiere a un estado particular de funcionamientos que comienza en la infancia, es multidimensional y está afectado positivamente por los apoyos individualizados.” (p.16). En concordancia con el autor la discapacidad intelectual es adaptativa y se presenta desde la infancia, manifestando limitaciones en el desarrollo de habilidades y destrezas del niño, presentando dificultades para interactuar con el entorno, sin embargo, no es una discapacidad permanente.

Siendo así, el ser humano es un ser activo por naturaleza que ocupa su tiempo en actividades encaminadas a responder sus necesidades y deseos, las personas se realizan a través de sus ocupaciones en interacción constante con el ambiente, de ahí la importancia de la realización de actividades que sean significativas y gratificantes para ellas. La salud y la ocupación están vinculadas porque la pérdida de salud disminuye la capacidad para comprometerse en la actividad, en este caso, apunta Miralles y Romero (2011), deberemos de adaptar la actividad teniendo en cuenta las características individuales de cada caso, sus motivaciones, gustos e intereses. Las actividades de la vida diaria (AVD) son un conjunto de tareas o conductas que una persona realiza de forma diaria y que le permiten vivir de forma autónoma e integrada en su entorno y cumplir su rol o roles dentro de la sociedad.

El ser humano presenta tres estructuras tridimensionales las cuales se ven afectadas cuando existen limitaciones, estas son: las funciones y estructuras del cuerpo, actividades personales y la participación. Cuando se ve afectada las funciones y estructuras del cuerpo se ven reflejadas en daños del organismo independientemente de la causa.

Cuando existe deficiencia en las estructuras y funciones del cuerpo, estas se manifiestan en las actividades y relaciones interpersonales, en el caso de niños con déficit intelectual existe una alteración en la estructura del sistema nervioso y se ve reflejado en la incapacidad mental para adaptarse al medio. Las actividades personales se manifiestan relacionadas en el desempeño ocupacional del individuo durante las tareas de la vida cotidiana, los infantes con déficit intelectual tienen dificultad en el momento de realizar la actividad y finalmente la participación es la manera en que interactúan las personas con el entorno y las restricciones que estos presentan. 
Cabe destacar, que la problemática de la discapacidad intelectual en el Ecuador se da debido a que el mayor porcentaje es de familias que se convierten en ayuda directa para la persona con Discapacidad en lo que en actividades de la vida diaria y productividad se refiere, aun si el individuo que posee la condición pudiera realizarlo de manera adecuada o no, razón principal por la que en este artículo, se evaluó la identidad y competencias ocupacionales en usuarios de la fundación con discapacidad intelectual leve.

\section{Desarrollo}

El ser humano presenta tres estructuras tridimensionales las cuales se ven afectadas cuando existen limitaciones, estas son: las funciones y estructuras del cuerpo, actividades personales y la participación. Cuando se ve afectada las funciones y estructuras del cuerpo se ven reflejadas en daños del organismo independientemente de la causa.

Cuando existe deficiencia en las estructuras y funciones del cuerpo, expresa Gento, S (2011), estas se manifiestan en las actividades y relaciones interpersonales, en el caso de niños con déficit intelectual existe una alteración en la estructura del sistema nervioso y se ve reflejado en la incapacidad mental para adaptarse al medio. Las actividades personales, explica Willard y Spackman. (2011), se manifiestan relacionadas en el desempeño ocupacional del individuo durante las tareas de la vida cotidiana, los infantes con déficit intelectual tienen dificultad en el momento de realizar la actividad y finalmente la participación es la manera en que interactúan las personas con el entorno y las restricciones que estos presentan.

Causas de la discapacidad intelectual. Es imposible determinar una causa precisa de la discapacidad intelectual se debe a varios trastornos biológicos y psicosociales, cabe recalcar que existen trastornos patológicos que llevan a una discapacidad intelectual antes de los 18 años de edad en el individuo. Al respecto, Fernández (2015), expone que las causas predisponentes se deben a la edad parenteral (alta incidencia de deficientes mentales en mujeres de edad superior a 35 años); consanguinidad (genes recesivos); factores socioeconómicos desfavorables (escasa estimulación sensorial); factores nutricionales (en edades muy tempranas); y el factor sexo (se da mayor incidencia en el sexo masculino). (P.78) El mismo autor, explica que las causas determinantes son aquellas que por sí solas pueden determinar la existencia del déficit y su grado. 
Se distinguen las siguientes categorías; deficiencia mental debida a infección, a agentes tóxicos, a traumatismo, a desordenes metabólicos, a aberraciones cromosómicas, a neoformaciones y tumores. En concordancia con el autor se puede identificar las causas de manera directa cuando se conoce el diagnóstico de enfermedades que derivan a discapacidades intelectuales, estas causas muchas veces se dan después del nacimiento e incluso pueden ser ocasionados por traumatismos físicos. A pesar de que existan determinantes de posibles causales aún existen niños con discapacidad intelectual que no presentan diagnostico etiológico.

Manifestaciones clínicas la discapacidad intelectual. Se puede presentar en diferente grado de afectación el déficit intelectual en las distintas áreas: cognitiva, motora, del lenguaje y social familiar. En el área cognitiva se presentan déficit de atención, memoria y concentración, pero en mayor cuantía en la recepción de la información y en el procesamiento. Las personas con déficit de atención tienen dificultades en el aprendizaje debido a que solo pueden receptar la información por una vía, sea visual o auditiva, por tanto se ve dificultada la respuesta.

En el área motora existe la presencia de la edad biológica y cronológica, se puede diferenciar en peso, estatura correspondiente a la edad cronológica. La adquisición de habilidades y destrezas se dan con el tiempo, existe deficiencia en motricidad fina y gruesa llevando a una descoordinación motora en algunos individuos.

En el área del lenguaje existen dificultades debido a las deficiencias en algunos órganos fonatorios, esto lleva a presentar déficit en la comunicación mediante la articulación de palabras, vocabulario limitado y uso de construcciones gramaticales simples. También existen déficits en habilidades no verbales como gestos, contacto ocular etc.

En el área social y familiar existe incapacidad de poder establecer relaciones interpersonales, afectando su parte emocional y muchas veces llevando al aislamiento del individuo. Si no existe aceptación por parte del entorno en el que se desenvuelve el individuo, se verá dificultado en adaptarse y optimizar sus capacidades. 


\section{Metodología}

La metodología del estudio se presentó con un enfoque cuali-cuantitativo apoyado en un proyecto viable y factible de tipo descriptivo experimental y de corte transversal. Para la obtención de la información se utilizó un instrumento tipo cuestionario de medición para las escalas de identidad ocupacional y de competencia ocupacional contentivo de cuatro alternativas de selección y 20 reactivos. La población era de 46 personas, y la muestra fue de 32 usuarios con discapacidad intelectual leve que asisten a la fundación Sergio Plaza Aguas, quienes recibieron talleres ocupacionales direccionados a fortalecer su identidad y competencias ocupacionales en pro de su desarrollo personal. El plan desarrollado, durante la intervención siguió los siguientes parámetros:

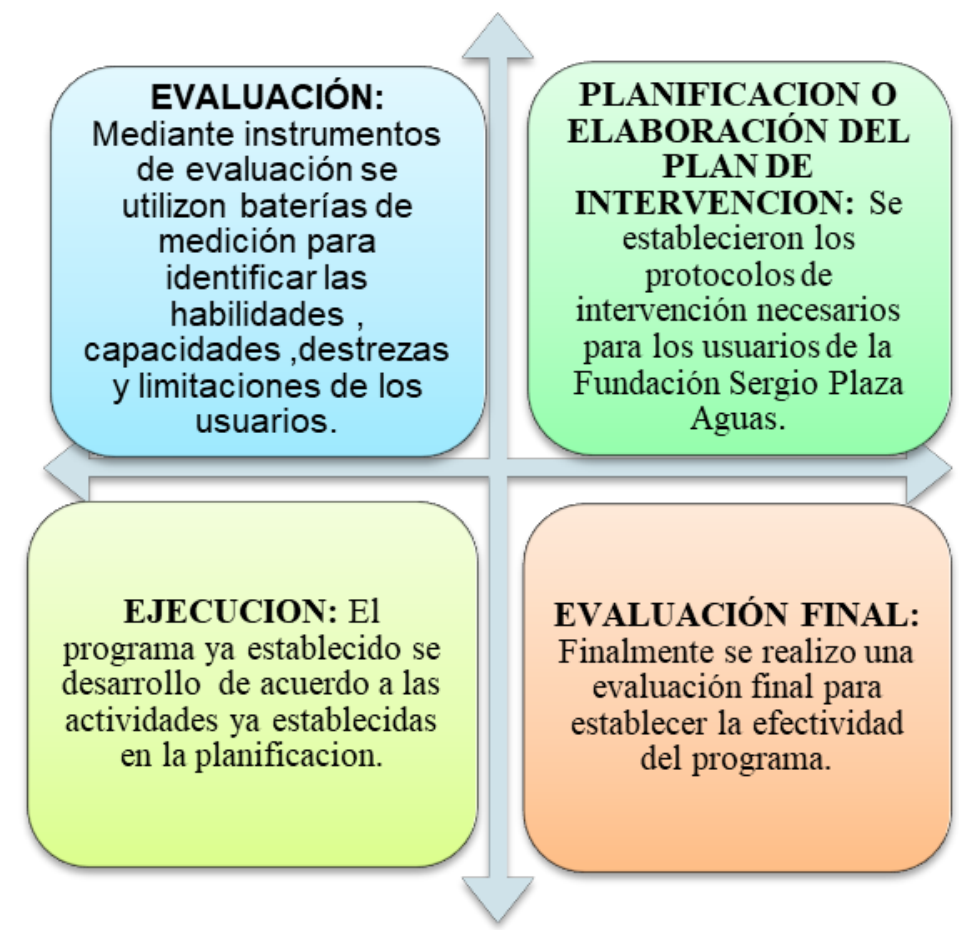

Fuente: Elaboración propia.

Para el análisis de la información se utilizó la hermenéutica como medio de interpretación de los datos obtenidos. 


\section{Resultados}

Previo a la aplicación del plan de intervención ocupacional, se pudo constatar que de una población total de 46 personas, el $15 \%$ se refirieron a 7 personas autistas, 1 Asperger que corresponde a 2\%, 4 personas con Discapacidad Visual con 9\%, 2 personas sordo-mudas con 4\% y finalmente 32 usuarios con Discapacidad intelectual leve con $70 \%$ del total de la población, estas últimas recibieron el plan de intervención.

En la tabla y grafico 1, se indican que del total de los usuarios con discapacidad intelectual leve que asisten a la Fundación Sergio Plaza Aguas, 15 usuarios se encuentran entre edades de 14-17 años y corresponde al 47\%, 9 personas pertenecen a edades entre 18 y 20 años correspondientes al $28 \%$ y finalmente 8 personas con edades entre 21 y 24 que corresponde al $25 \%$.

Tabla 1. Muestra por edad

\begin{tabular}{r|c|c|c}
\multicolumn{1}{c|}{ ITEMS DETALLE } & \multicolumn{2}{c}{ FRECUENCIA } & PORCENTAJE \\
\hline $\mathbf{1}$ & $14-17$ & 15 & $47 \%$ \\
\hline $\mathbf{2}$ & $18-20$ & 9 & $28 \%$ \\
\hline $\mathbf{3}$ & $21-24$ & 8 & $25 \%$ \\
\hline $\mathbf{4}$ & TOTAL & 32 & $100 \%$ \\
\hline
\end{tabular}

Fuente: elaboración propia

1 14-17 $\quad 218-20 \quad 321-25$

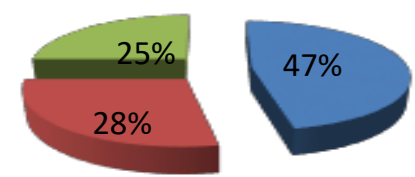

Gráfico 1. Muestra por edad 
En la tabla y grafico 2, se observa que del total de la muestra por género, en la que se indica que 11 personas son del sexo masculino que corresponde al $34 \%$ y 21 personas de sexo femenino que se corresponden al $66 \%$ del total de la muestra establecida.

Tabla 2. Muestra por género

\begin{tabular}{|c|c|c|c|}
\hline ITEMS & DETALLE & FRECUENCIA & PORCENTAJE \\
\hline $\mathbf{1}$ & MASCULINO & 11 & $34 \%$ \\
\hline $\mathbf{2}$ & FEMENINO & 21 & $66 \%$ \\
\hline $\mathbf{3}$ & TOTAL & 32 & $100 \%$ \\
\hline
\end{tabular}

Fuente: elaboración propia

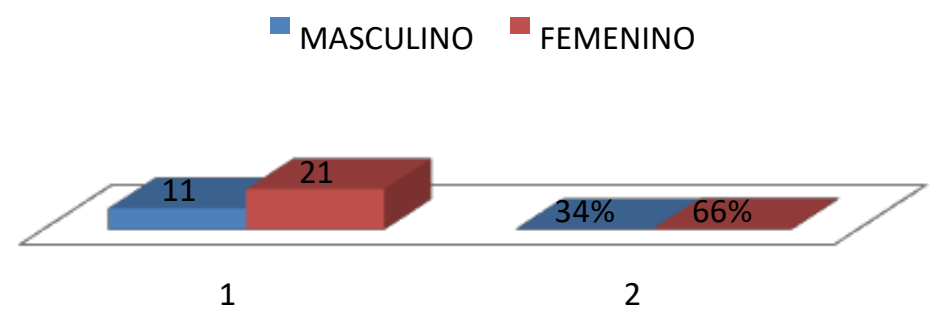

Gráfico 2. Muestra por género

La tabla y grafico 3, presenta la escala de identidad ocupacional, en la que constato inicialmente que 7 personas presentaron problemas para identificar interés por alguna ocupación con 22\%; el $25 \%$ por igual presentaron algunos problemas y/o con una identidad ocupacional competente y el $28 \%$ alcanzaron a evaluarse con una identidad ocupacional de satisfactorio. En la evaluación final se observa 5 personas con problemas para identificar interés por alguna ocupación con 16\%, 6 con algunos problemas representados en el 19\%, 14 personas con identidad ocupacional satisfactoria con el $44 \%$ y 7 usuarios con competentes competencias ocupacionales representados en el $22 \%$ del total de los investigados. 
Tabla 3. Escala de identidad ocupacional

\begin{tabular}{|l|r|r|r|r|}
\hline $\begin{array}{l}\text { IDENTIDAD } \\
\text { OCUPACIONAL }\end{array}$ & \multicolumn{2}{|c|}{ INICIO } & \multicolumn{2}{c|}{ FINAL } \\
\hline DETALLE & FRECUENCIA & PORCENTAJE & FRECUENCIA & PORCENTAJE \\
\hline PROBLEMA & 7 & $22 \%$ & 5 & $16 \%$ \\
\hline ALGUNOS PROBLEMAS & 8 & $25 \%$ & 6 & $19 \%$ \\
\hline SATISFACTORIO & 9 & $28 \%$ & 14 & $44 \%$ \\
\hline COMPETENTE & 8 & $25 \%$ & 7 & $22 \%$ \\
\hline TOTAL & 32 & $100 \%$ & 32 & $100 \%$ \\
\hline
\end{tabular}

Fuente: elaboración propia

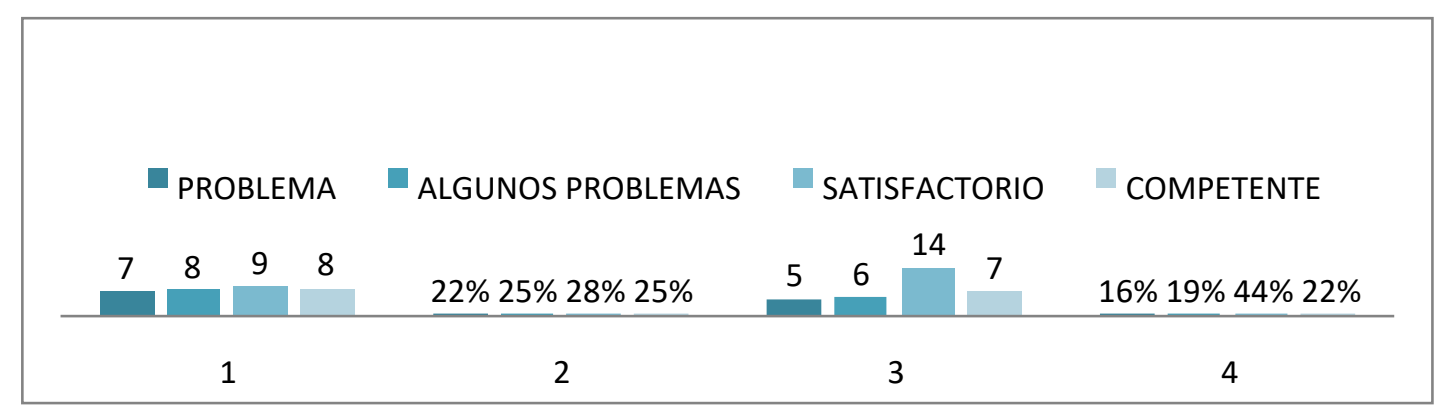

Gráfico 3. Escala de identidad ocupacional

En la tabla y grafico 4, presenta los resultados de la evaluación de las competencias iniciales y finales entre los usuarios investigados. En la misma, se indica que de las 32 personas con discapacidad intelectual leve evaluadas inicialmente, se encontraron 7 personas con problemas para realizar alguna ocupación representando en el 22\%; el 25\% con algunos problemas y con competencias ocupacionales satisfactorias y el 28\% competentes. Para la evaluación final, se pudo constatar que solo el $16 \%$ presento problemas o algún problema, el $38 \%$ logro evaluarse con competencias ocupacionales satisfactorios y el $31 \%$ develaron competentes competencias ocupacionales. 
Tabla 4. Escala de competencias ocupacionales

\begin{tabular}{|l|r|r|r|r|}
\hline $\begin{array}{l}\text { COMPETENCIAS } \\
\text { OCUPACIONALES }\end{array}$ & \multicolumn{2}{|c|}{ INICIO } & \multicolumn{2}{c|}{ FINAL } \\
\hline DETALLE & FRECUENCIA & PORCENTAJE & FRECUENCIA & PORCENTAJE \\
\hline PROBLEMA & 7 & $22 \%$ & 5 & $16 \%$ \\
\hline $\begin{array}{l}\text { ALGUNOS } \\
\text { PROBLEMAS }\end{array}$ & 8 & $25 \%$ & 5 & $16 \%$ \\
\hline SATISFACTORIO & & & & $38 \%$ \\
\hline COMPETENTE & 8 & $25 \%$ & 12 & $31 \%$ \\
\hline TOTAL & 9 & $28 \%$ & 10 & $100 \%$ \\
\hline
\end{tabular}

Fuente: elaboración propia

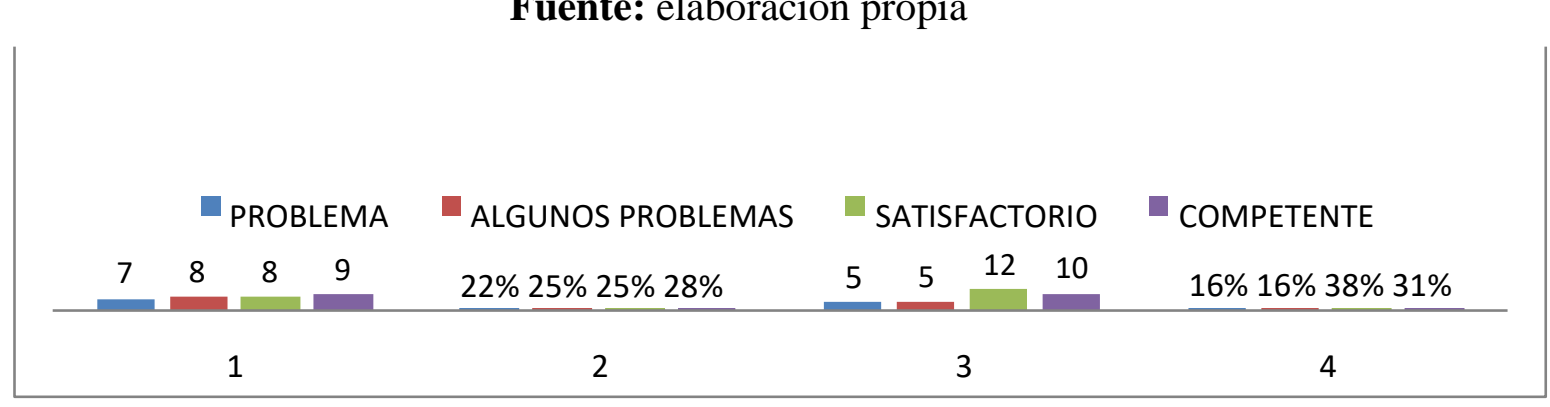

Grafico 4. Escala de competencias ocupacionales

\section{Conclusiones}

-El 70\% del total de la población de usuarios que asisten a la fundación Sergio Plaza Aguas, presentan Discapacidad Intelectual leve.

-El mayor porcentaje de los usuarios con discapacidad intelectual leve se corresponden a edades comprendidas entre 14-17 años y el menor porcentaje se presentó en usuarios cuyas edades oscilan entre 21 y 24.

-El género de mayor predominio entre los usuarios es del sexo femenino que se corresponden al $66 \%$ del total de la muestra establecida.

-Se constató posterior a los resultados de la aplicación del instrumento de evaluación final a los usuarios investigados, mejoría en comparación a la evaluación inicial, manifiesto en la presentaron inicial de problemas y algunos problemas para identificarse con interés por alguna ocupación al disminuir su porcentaje. Asimismo, la identificación de interés por alguna ocupación en referencia a la evaluación de satisfactoria o competente mejoró durante la 
evaluación final al presentar mayor porcentaje, en tanto que pasó de un $28 \%$ a un $44 \%$ en el resultado satisfactorio.

-Fue posible corroborar posterior a los resultados de la aplicación del instrumento de evaluación final a los usuarios investigados, mejoría en comparación a la evaluación inicial, manifiesto en la presentaron inicial de problemas y algunos problemas para realizar alguna ocupación al disminuir su porcentaje. Asimismo, la realización de alguna ocupación en referencia a la evaluación de satisfactoria o competente mejoró durante la evaluación final al presentar mayor porcentaje.

\section{Referencias Bibliográficas}

Bassedas, E y Bales (2010). Alumnado con discapacidad intelectual y retraso del desarrollo.

\section{España. Ediciones GRAO.}

Fernández, J. (2015). Atención a la diversidad en el aula de educación infantil. España. Colección: didáctica y desarrollo. Ediciones paraninfo.

Gento, S (2011). Tratamiento educativo de la diversidad en personas con discapacidad intelectual. España. Editorial UNED.

Miralles, P y Romero, D. (2011). Actividades de la vida diaria masson. Barcelona. España. Willard y Spackman. (2011). Terapia ocupacional. España. 11a edición. Editorial medica panamericana. 